\title{
Bemerkungen zu dem Enkomion des Joseph Studites auf den heiligen Demetrios (BHG 535)
}

\author{
SOFIA KOTZABASSI
}

Ein erster Aufenthalt des Joseph Studites in Thessaloniki ist durch den Brief seines Bruders Theodoros an ihren Onkel Platon, Abt des Sakkudion-Klosters, den der Jubilar in seiner monumentalen Ausgabe ediert hat, bezeugt. ${ }^{1}$ In diesem Brief beschreibt Theodoros Studites die Reise nach Thessaloniki und ihre Erlebnisse von den ersten Tagen in der Stadt, in die er und sein jüngerer Bruder Joseph im Jahre 797 verbannt wurden. Dank der Großzügigkeit des Statthalters durften beide Brüder nicht nur von dem Metropoliten empfangen werden, sondern auch die Kirche der Hagia Sophia und die Basilika des Schutzpatrons von Thessaloniki, des heiligen Demetrios, besuchen, bevor sie eingesperrt wurden. ${ }^{2}$

Während dieser Besuch in Thessaloniki der einzige für Theodoros war, kam Joseph als Metropolit nach einigen Jahren wieder in die Stadt. Wenig ist bekannt über seine kurze Amtszeit, doch kennen wir mindestens eine Rede, ${ }^{3}$ die er in der Kirche des heiligen Demetrios an seinem Festtag gehalten hat. ${ }^{4}$

1 Theodori Studitae Epistulae, rec. G. Fatouros (CFHB XXX/1-2 - Series Berolinensis), Berlin/New York 1992, 146* und 11-16 (Brief Nr. 3). Zum Leben des Joseph Studites s. die zusammenfassende Darstellung in der PmbZ, Nr. 3448.

2 Theodori Studitae Epistulae (s. Anm. 1), 15.99-122. Der Besuch fand am Montag, den 27. März 797 statt.

3 S. Fr. Halkin, Bibliotheca Hagiographica Graeca (SubHag 8a), Bruxelles 1958, Nr. 535. Das Enkomion wurde im Jahre 1889 in Moskau vom Archimandriten Arsenios aus dem Kodex Mosqu. GIM 162 (Vlad. 380) veröffentlicht; leider konnte ich diese Ausgabe, trotz langjähriger Bemühungen, nicht ausfindig machen. Im Folgenden wird der Text des Enkomions nach dem Mosqu. GIM 162 (Vlad. 380) zitiert, wenn es nicht anders angegeben ist.

4 Dies wird an einigen Ausdrücken in der Rede dokumentiert, wie z.B. am Anfang des Enkomi-

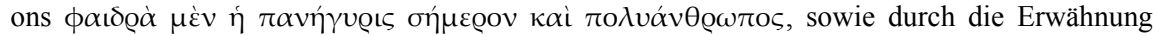

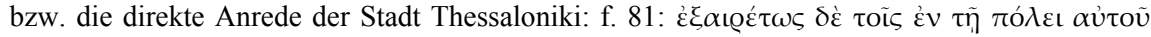

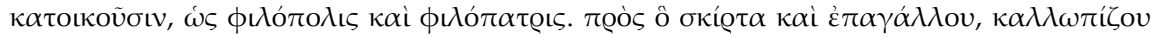

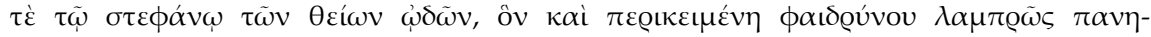

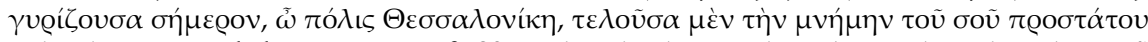

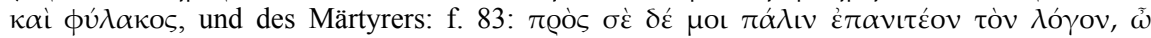

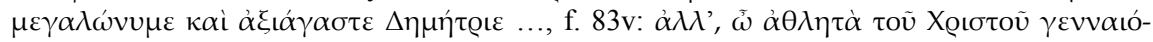

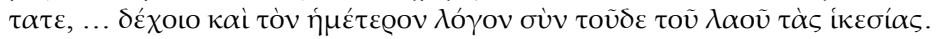


Joseph kam im Jahre 807 auf den Metropolitenthron von Thessaloniki, blieb aber nur zwei Jahre und wurde dann wegen des Streits der Studiten mit dem Kaiser für die Wiedereinsetzung des Oikonomos Joseph abgesezt und verbannt. ${ }^{5}$ Der Tod des Kaisers Nikephoros I. und die Erhebung Michaels I. zum Souverän signalisieren die Rückkehr der Studiten nach Konstantinopel und Josephs nach Thessaloniki, die wahrscheinlich im Jahre 811 stattfand. ${ }^{6}$ Sein Widerstand gegen die ikonoklastische Politik des Leon V. hatte seine erneute Absetzung und Verbannung im Jahre 815 zur Folge. $^{7}$

Das Enkomion des Joseph Studites ist das zweitälteste von allen erhaltenen, die auf den Schutzpatron von Thessaloniki verfasst wurden. Joseph geht nicht auf die Einzelheiten des Martyriums, wie wir es von den späteren Enkomia gewöhnt sind, ein und lässt sich nicht von seinem Vorgänger Johannes ${ }^{8}$ beeinflussen, sondern konzentriert sich in seinem Enkomion auf das Motiv der Verehrung der Märtyrer durch die Imitierung ihres Lebens.

Den rhetorischen Charakter des Textes betonen zahlreiche rhetorische Fragen, Asyndeta und weitere rhetorische Figuren, die Joseph verwendet. ${ }^{9}$ Joseph Studites zitiert oft aus dem Neuen Testament, ${ }^{10}$ während er zweimal aus der Rede des Gregor von Nazianz auf Gregor von Nyssa (or. 11) schöpft: ${ }^{11}$

5 Die Absetzung von Joseph folgt nach einem Treffen mit dem Logothetes tou Dromou, von dem Theodoros Studites in einem Brief an die Sakkudion-Mönche berichtet; vgl. Theodori Studitae Epistulae (s. Anm. 1), 85.11-21 (Brief 31) und 172*-173*.

6 Das genaue Datum seiner Rückkehr ist ungewiss; s. G. Fedalto, Hierarchia Ecclesiae Constantinopolitanae: series episcoporum ecclesiarum christianarum orientalium, Padua 1988, I, 424.

7 S. PmbZ, Nr. 3448 (401). Zu Leon V. und zu seiner ikonoklastischen Politik s. D. Turner, The Origins and Accession of Leo V (813-820), in: JÖB 40 (1990) 171-203 und Th. K. Korres,

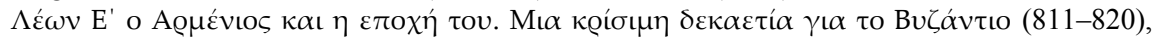
Thessaloniki 1996, 78-79.

8 Sein Enkomion ist von A. Philippidis-Braat, L'encômion de saint Démétrius par Jean de Thessalonique, in: TM 8 (1981) 397-414, publiziert.

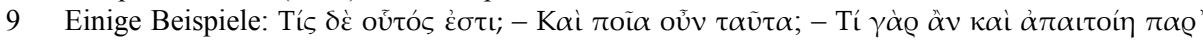

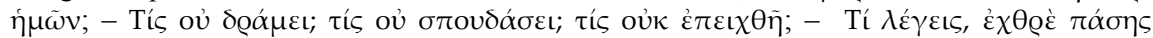

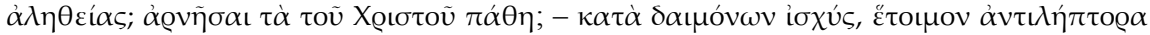

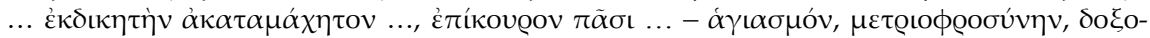

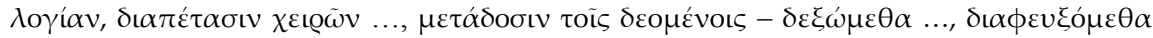

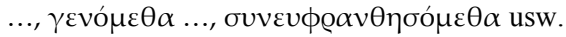

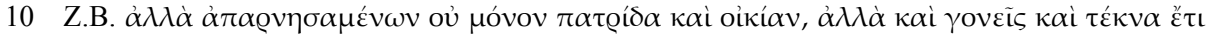

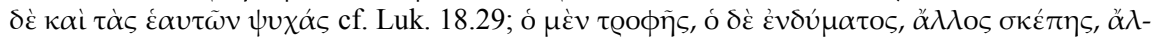

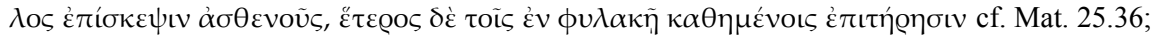

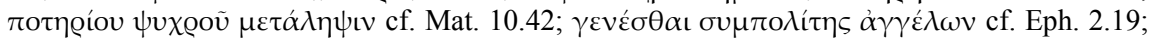

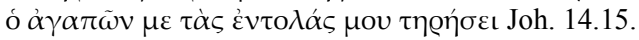

11 S. M.-A. Calvet-Sebasti, Grégoire de Nazianze. Discours 6-12 (SC 405), Paris 1995, 328-346. 


\begin{tabular}{|c|c|}
\hline $\begin{array}{c}\text { Gregor von Nazianz, or. } 11 \text { (ed. } \\
\text { Calvet-Sebasti) }\end{array}$ & $\begin{array}{c}\text { Mosqu. GIM } 162 \text { ( Vlad. 380), } \\
\text { f. } 82\end{array}$ \\
\hline 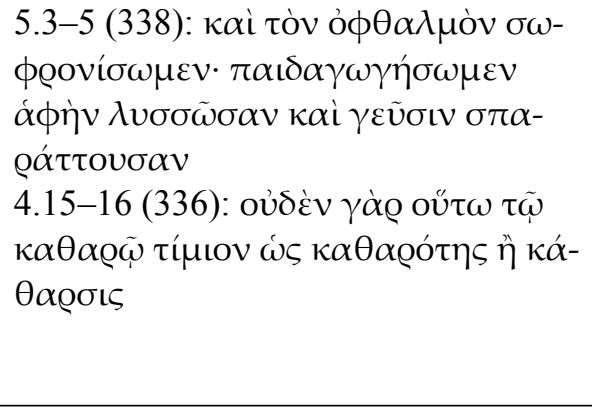 & 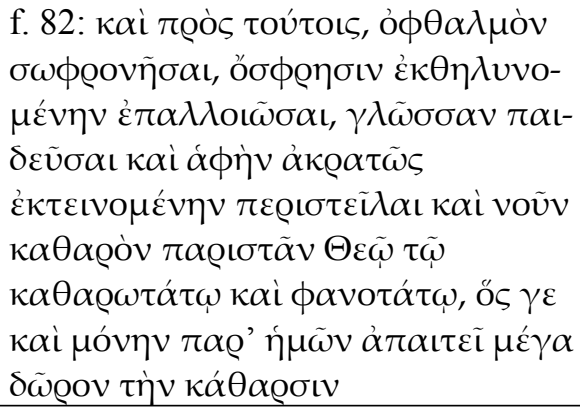 \\
\hline 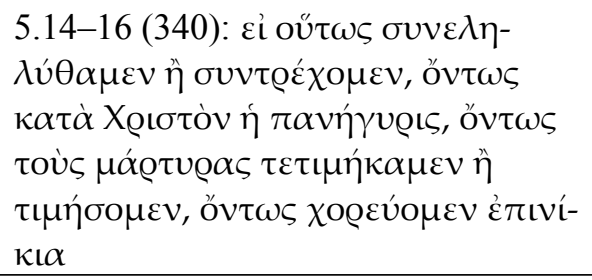 & 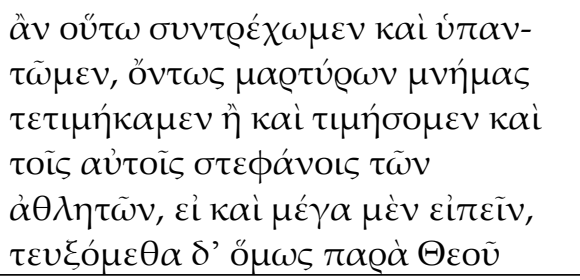 \\
\hline
\end{tabular}

Ungewöhnlich ist darüber hinaus das lange Zitat aus dem Hohen Lied, welches Joseph benutzte, um die äußeren Charakteristika des heiligen Demetrios darzustellen: ${ }^{12}$

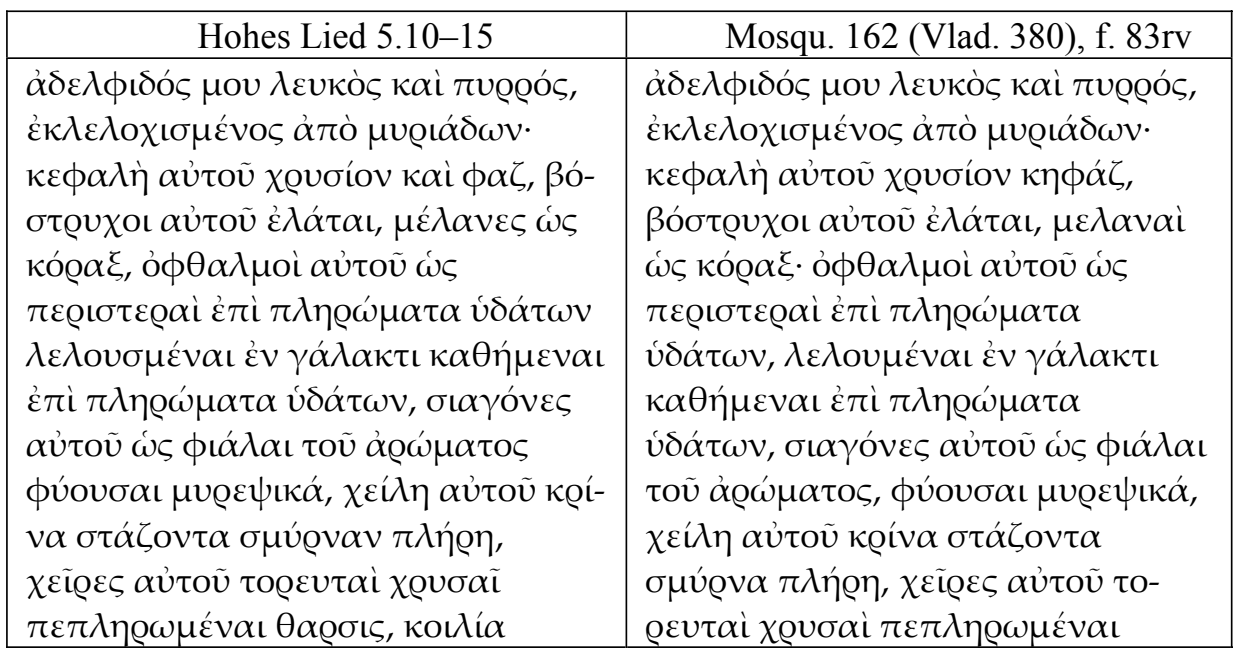

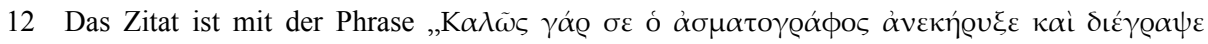
$\lambda \varepsilon \dot{\gamma} \omega v^{*}$ " eingeführt. 


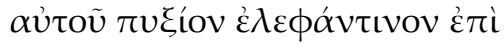

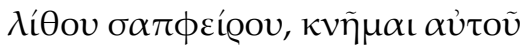

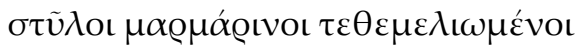

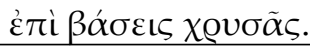

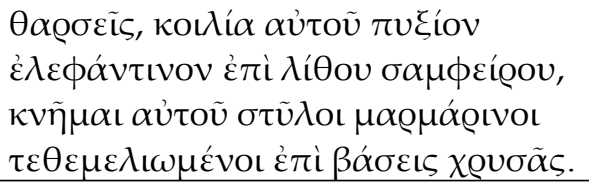

Die Rede ist in drei Kodizes überliefert: dem Mosqu. GIM 162 (Vlad. 380) aus dem Jahre $1021 / 22^{13}$ und dessen Apographon, dem Kodex Athous, Iberon 426 aus dem 16. Jh. ${ }^{14}$, sowie dem Kodex Paris. 1517 aus dem 12. Jh. ${ }^{15}$

Während der Mosqu. GIM 162 (Vlad. 380) eine hagiographische Sammlung für das ganze Jahr ist, stellt der Paris. 1517 eine Sammlung von hagiographischen Texten für den heiligen Demetrios dar ${ }^{16}$. Die Art der Abweichungen zwischen der Textversion, die jeder Kodex überliefert, schließt jede stemmatische Abhängigkeit des Paris. 1517 vom Mosqu. GIM 162 (Vlad. 380) aus und weist deutlich auf eine Bearbeitung des Textes hin. Einige Auslassungen und Umstellungen, die als Kopistenfehler hätten erklärt werden können, sprechen nicht gegen die Hypothese einer Bearbeitung; denn es stehen die Auslassungen meistens in Verbindung zu weiteren Abänderungen, die nicht als notwenige Eingriffe für die Wiederherstellung des Textes verstanden werden können. Die Palette dieser Änderungen er-

13 Der Kodex ist von Theophanes geschrieben worden und befand sind ursprünglich in der Bibliothek des athonitischen Iberon-Klosters. Für eine ausführliche Beschreibung s. Archimandrit Vladimir, Систематическое описаніе рукописей Москоской Синодальной (Патріаршей) Библиотеки. Часть первая: Рукописи греческія, Moskau 1894, 572-573 und ebenso A. Ehrhard, Überlieferung und Bestand der hagiographischen und homiletischen Literatur der griechischen Kirche, Leipzig/Berlin 1952, III 741-742; s. auch die gesammelte Bibliographie zum Kodex in B. Fonkić/Th. Poliakov, Греческие Рукописи Московской Синодальной Библиотеки. Палеографические, кодикологические и библиографические дополнения к каталогу архимандрита Владимира (филантропова), Moskau 1993, 124.

14 Aus Versehen ist dieser Kodex als Textzeuge für eine in der Tat nicht existierende Lobrede des Joseph Studites auf den Märtyrer Nestor sowohl bei Ehrhard (s. Anm. 13), III, 450 (Nr. 10) als auch in der Bibliotheca Hagiographica Graeca (s. Anm. 3), Nr. 2292 verzeichnet. Eine Beschreibung des Kodex s. S. P. Lambros, Catalogue of the Greek Manuscripts on Mount Athos, Cambridge 1900 (Ndr. Amsterdam 1966), II, 145 und Ehrhard (s. Anm. 13), III, 450. Das stemmatische Verhältnis des Kodex zu dem Mosqu. GIM 162 (Vlad. 380), welches sich durch die Kollation des Textes ergibt, wird auch durch die Provenienz seiner Vorlage aus dem Iberon-Kloster bestätigt.

15 Für eine Beschreibung des Kodex s. H. Omont, Inventaire sommaire des manuscrits grecs de la Bibliothèque Nationale et des autres bibliothèques des Paris et des Départements, Paris 1888 (Ndr. Hildesheim/Zürich/New York 2000), II, 75, Ehrhard (s. Anm. 13), III, 901, Fr. Halkin, Manuscrits grecs de Paris. Inventaire hagiographique (SubHag 44), Bruxelles 1968, 194, P. Lemerle, Les plus anciens recueils des miracles de saint Démétrius, I. Le texte, Paris 1979, 15-22 und Philippidis-Braat (s. Anm. 8), 397-398.

16 Der Kodex enthält beide Sammlungen der Wundertaten des heiligen Demetrios, das Enkomion von Johannes von Thessaloniki und Joseph Studites und zwei Homilien des Kaisers Leon VI. des Weisen auf den heiligen Demetrios; vgl. die Beschreibung des Kodex wie in Anm. 15. 
streckt sich von einfacher Ersetzung von Wörtern durch andere, ${ }^{17}$ Änderungen von finiten Verben durch Infinitive bzw. Partizipien und umgekehrt, ${ }^{18}$ bis zur vollständigen Umschreibung eines Satzes, wie die nachstehenden Beispiele dokumentieren. Diese Bearbeitung hängt wahrscheinlich mit der Aufnahme des Enkomions in die Sammlung der Texte über den heiligen Demetrios, die der Paris. 1517 präsentiert, zusammen. Einige Beispiele: ${ }^{19}$

\begin{tabular}{|c|c|}
\hline Mosqu. 162 (Vlad. 380) & Paris. 1517 \\
\hline 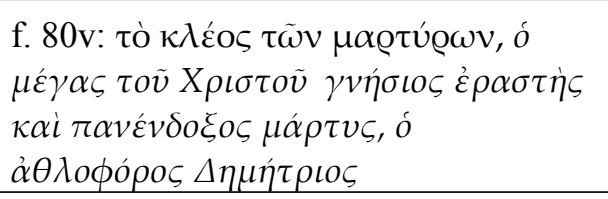 & 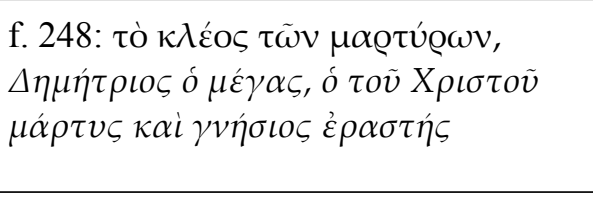 \\
\hline 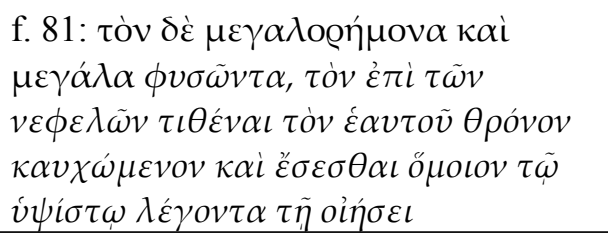 & 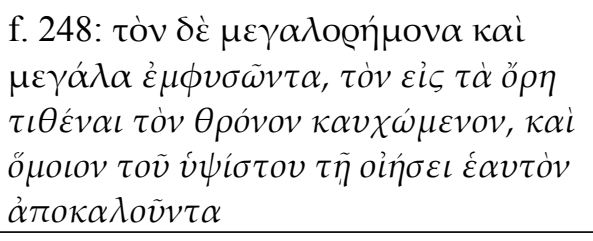 \\
\hline 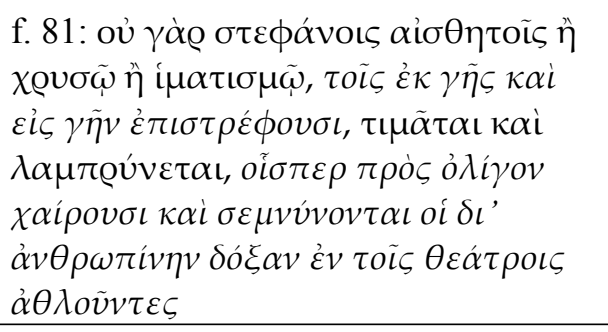 & 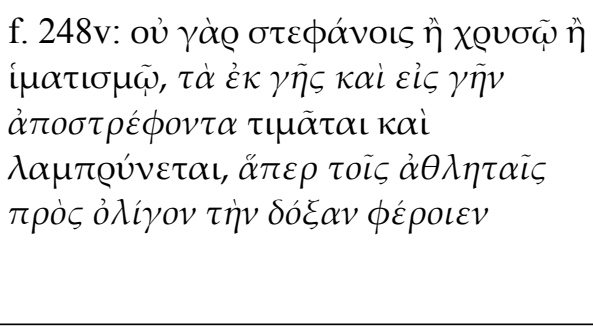 \\
\hline 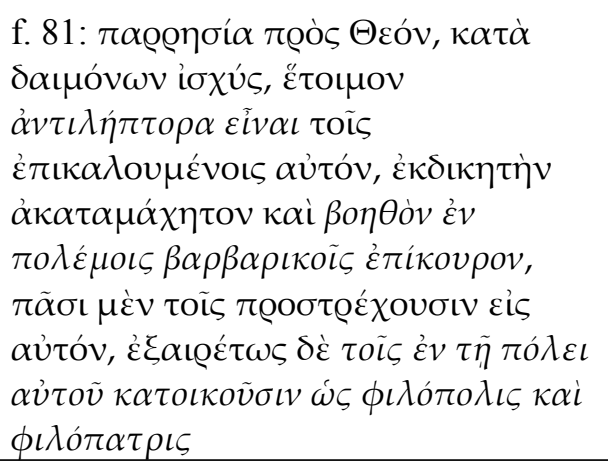 & 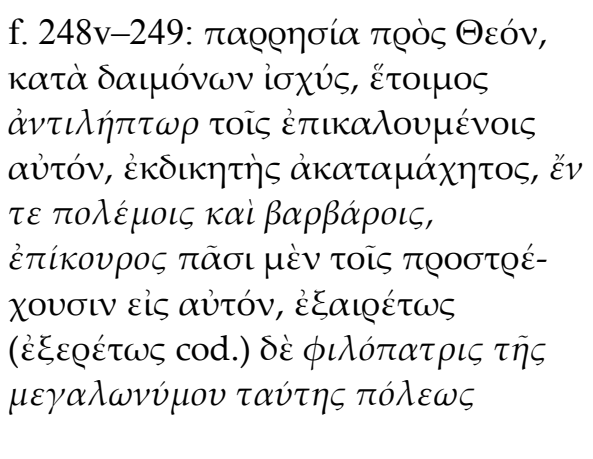 \\
\hline
\end{tabular}

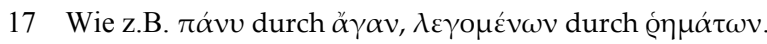

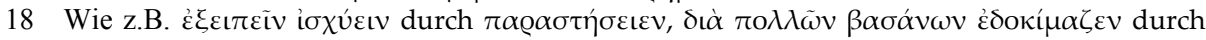

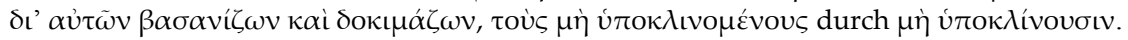

19 Meistens sind die Rechtschreibung und die Interpunktion der Handschriften beibehalten. 


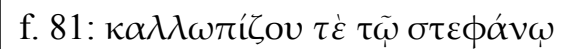

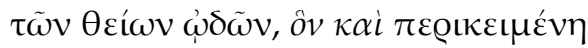
$\phi \alpha \iota \delta \rho v ́ v o v \lambda \alpha \mu \pi \rho \tilde{\omega} \varsigma \pi \alpha v \eta \gamma v Q i ́ \zeta o v-$

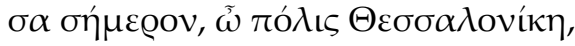

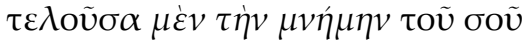

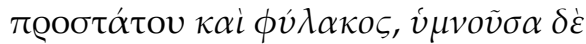

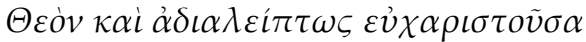

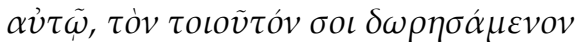

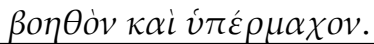

f. 81: $\dot{\alpha} \lambda \lambda^{\prime}$ oủd $\dot{\varepsilon} \pi \tilde{\alpha} \sigma \alpha \iota \gamma \lambda \tilde{\omega} \sigma \sigma \alpha \iota \tau \tilde{\omega} \nu$

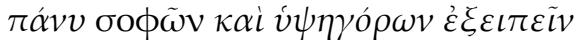

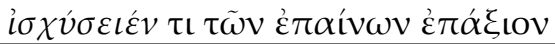

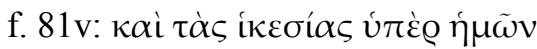

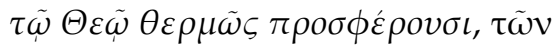
$\tau \mu \omega \hat{v} \tau \tau \omega \nu \alpha \hat{v} \tau \tilde{\omega} \nu \tau \dot{\alpha} \mu \nu \eta \mu o ́ \sigma v v \alpha \kappa \alpha \grave{i}$

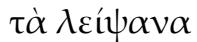

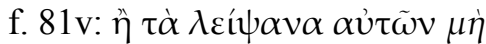

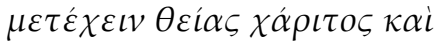

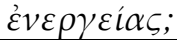

f. 81v: $\pi \lambda \eta \rho о \phi о \rho \eta \sigma \varepsilon \iota ~ \gamma \alpha \dot{\alpha} \rho \dot{\eta} \tilde{\alpha} \varsigma \dot{\eta}$

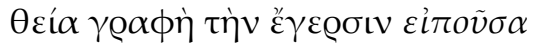

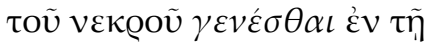

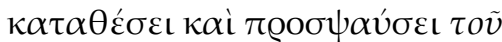

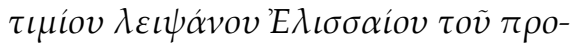

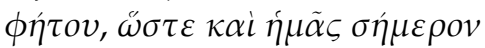

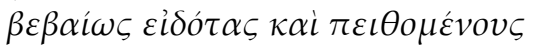
$\pi \circ \lambda \lambda \tilde{\eta} \varsigma \chi \alpha \dot{\alpha} \rho \iota \tau o \varsigma \alpha \dot{\xi} \xi \iota \tilde{v} \sigma \theta \alpha \iota \tau \dot{\alpha} \tau \tilde{\omega} v$

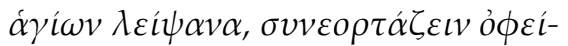

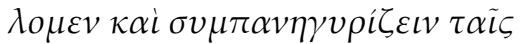

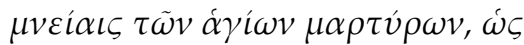

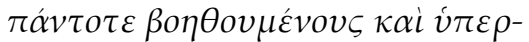

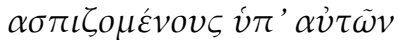

f. 81v: $\alpha \pi o ́ \delta \varepsilon \xi ı \zeta \delta \dot{\varepsilon} \tau \tilde{\omega} v \lambda \varepsilon \gamma o \mu \varepsilon \dot{v} v \omega v \dot{\eta}$

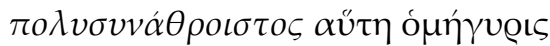

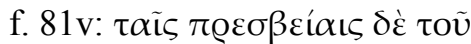

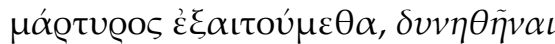

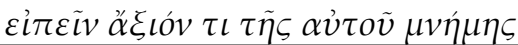

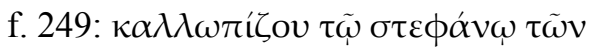
$\theta \varepsilon i \omega \omega \nu \omega \tilde{\omega} \nu \pi \varepsilon \varrho \iota \kappa \varepsilon \mu \varepsilon \dot{v} \eta$,

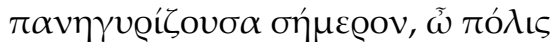
$\Theta \varepsilon \sigma \sigma \alpha \lambda$ ovík,$\tau \varepsilon \lambda$ ov $\sigma \alpha \tau \dot{\alpha}$

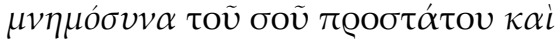
$\mu \alpha \dot{\alpha} \tau \tau \rho \rho \varsigma$.

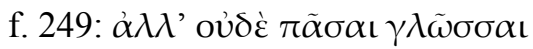

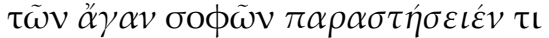

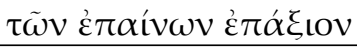

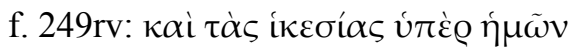
$\tau \tilde{\omega} \nu \tau \iota \mu \omega \dot{\nu} \tau \omega \omega \nu \alpha \dot{v} \tau \tilde{\omega} \nu \tau \dot{\alpha}$

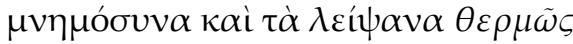
$\pi \rho 0 \sigma \phi \varepsilon \dot{\varepsilon} \rho 0 v \sigma \iota \mathrm{V}$

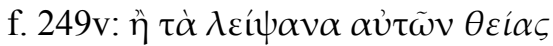

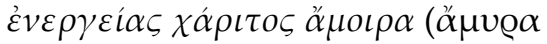
cod.) $\varepsilon \dot{i v} \alpha \iota \dot{\alpha} \pi \varepsilon \phi \theta \dot{\varepsilon} \gamma \xi \alpha \tau o$;

f. 249v: $\tilde{\alpha} \rho \alpha \pi \varepsilon \pi \lambda \dot{\alpha} v \eta \kappa \varepsilon v \eta \dot{\eta} \mu \tilde{\alpha} \bar{\eta}$

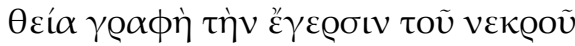

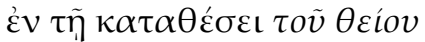

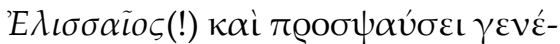

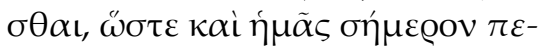

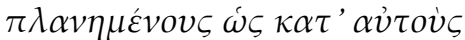
$\dot{\varepsilon} \kappa \varepsilon \dot{i v o v \varsigma} \sigma v v \varepsilon o \rho \tau \alpha \dot{\zeta} \zeta \iota \iota \mu \eta \delta \dot{\varepsilon} v \dot{\varepsilon} \kappa \tau \tilde{\omega} v$

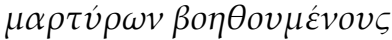

f. $249 \mathrm{v}-250: \dot{\alpha} \pi o ́ \delta \varepsilon \xi \xi \varsigma \delta \dot{\varepsilon} \tau \tilde{\omega} \nu$

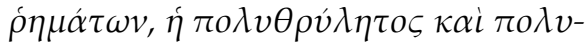

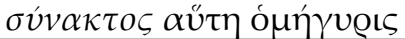

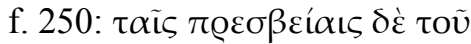

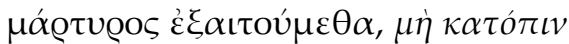
$\dot{\varepsilon} \lambda \theta \varepsilon \tilde{\imath} v \tau \alpha \tilde{\iota} \varsigma \tau \tilde{\omega} v \dot{\alpha} \theta \lambda \eta \tau \tilde{\omega} v \mu v \eta \mu \alpha \iota \varsigma$ 


\begin{tabular}{|c|c|}
\hline 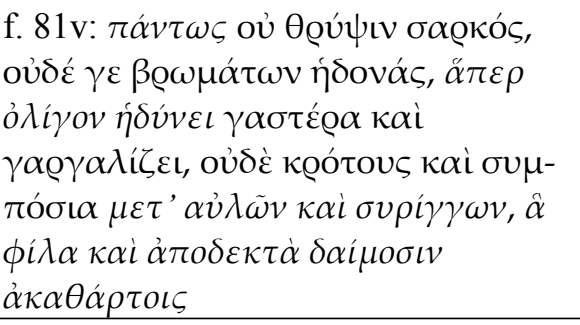 & 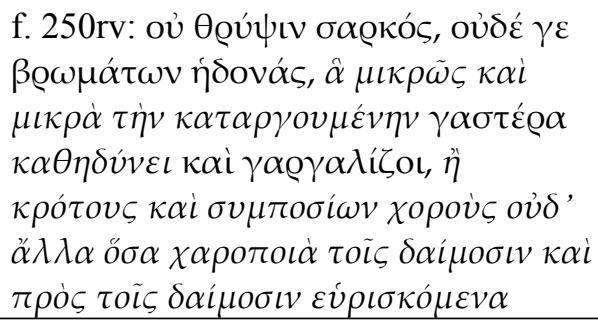 \\
\hline 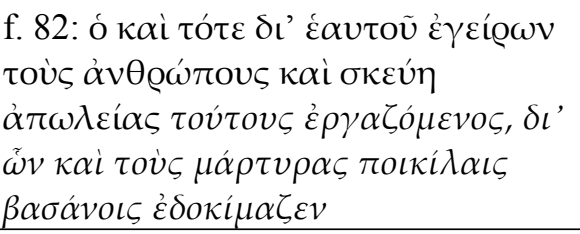 & 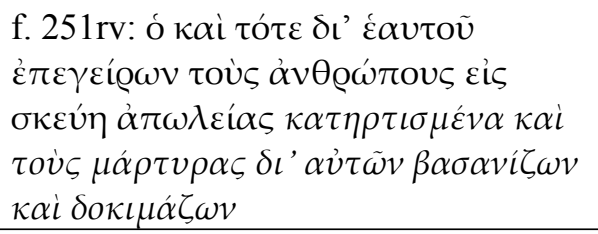 \\
\hline 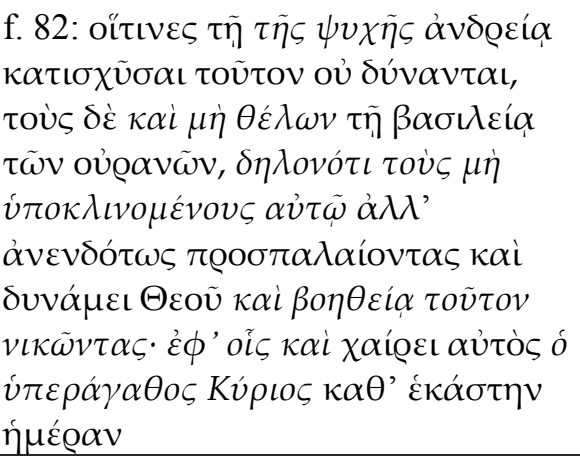 & 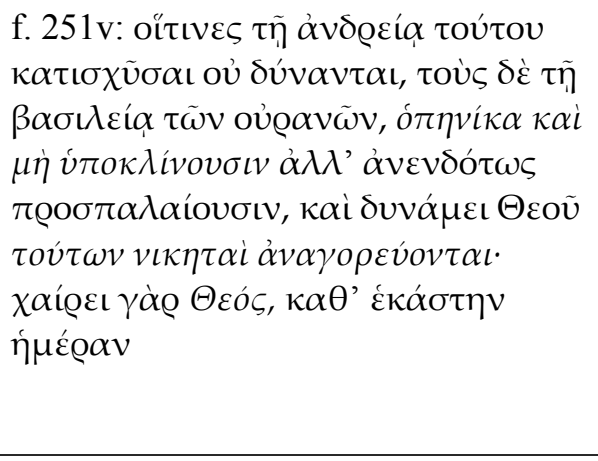 \\
\hline 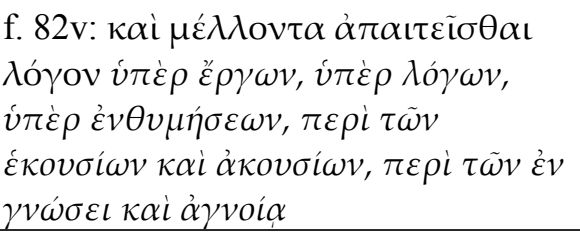 & 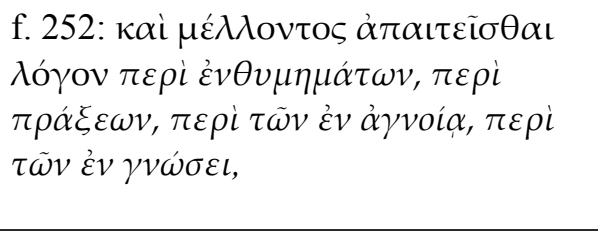 \\
\hline 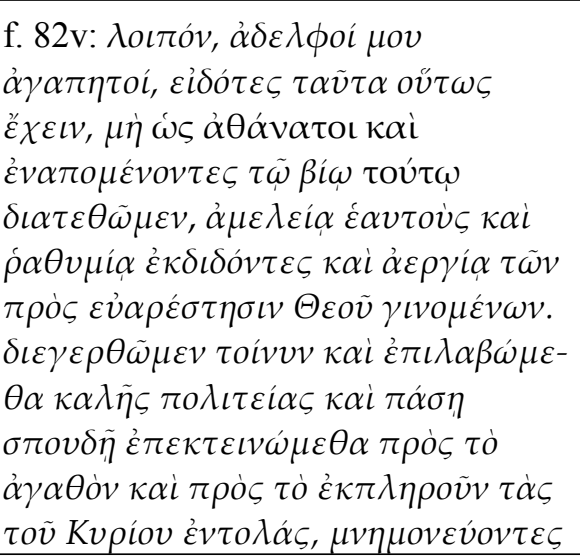 & 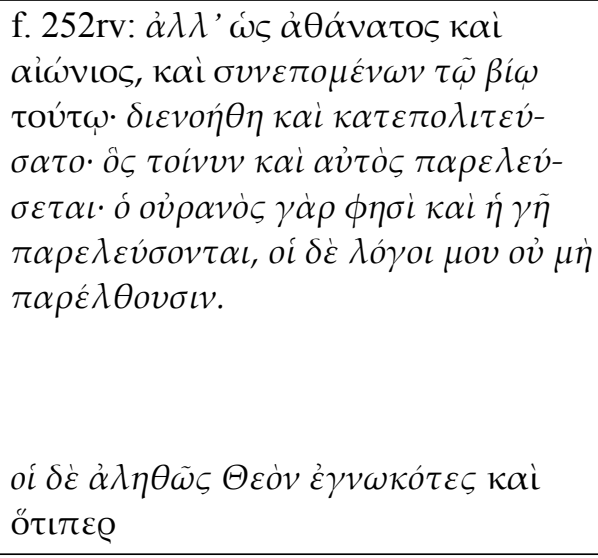 \\
\hline
\end{tabular}




\begin{tabular}{|c|c|}
\hline 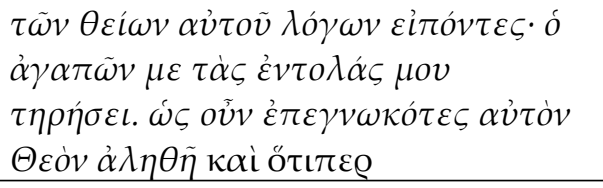 & \\
\hline 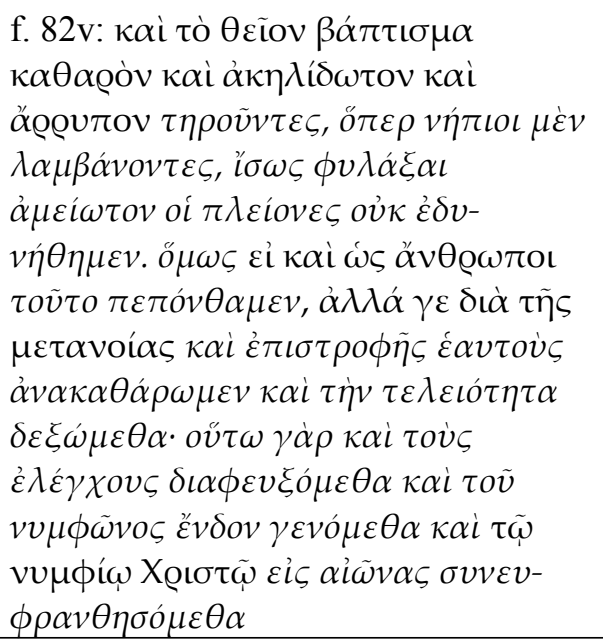 & 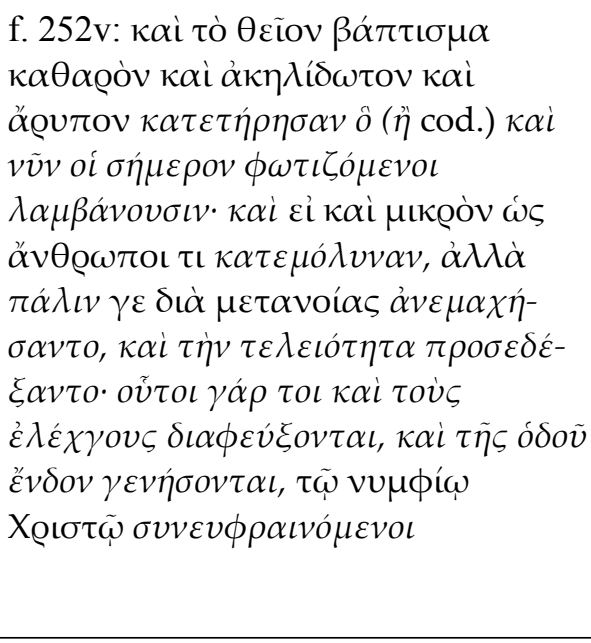 \\
\hline 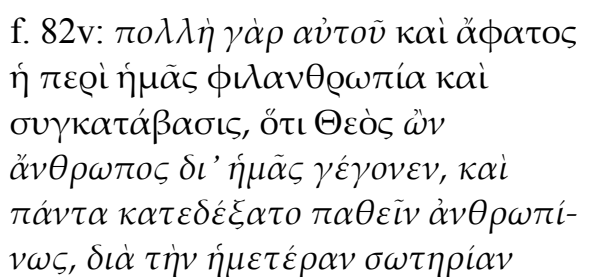 & 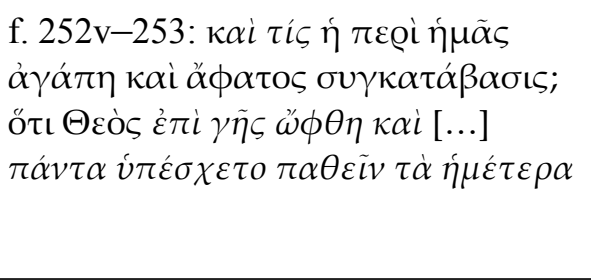 \\
\hline
\end{tabular}

Beachtenswert sind die Abweichungen beider Handschriften an zwei Stellen: Im ersten Fall überliefert der Mosquensis den Text folgendermaßen:

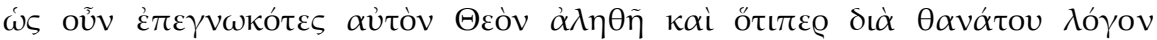

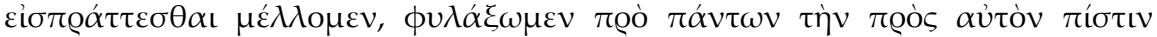

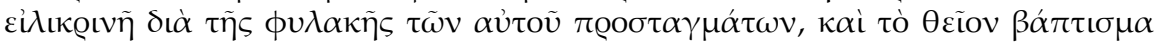

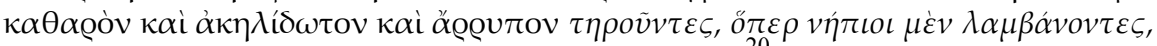

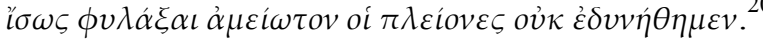

Joseph fordert seine Hörer auf, die Taufe, der man sich als Kind unterzog, rein, keusch und unbefleckt zu bewahren. Der Parisinus bietet eine ziemlich abweichende Formulierung, besonders wo Joseph über die Taufe spricht: 


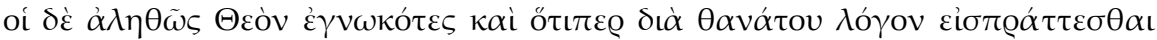

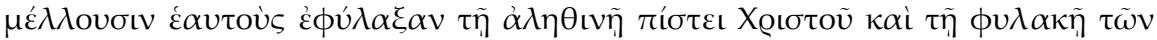

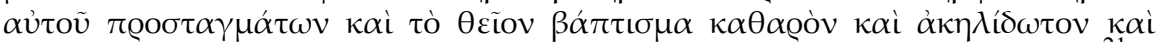

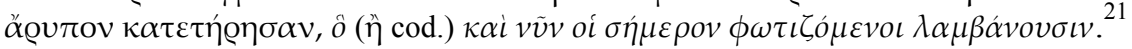

Der Bearbeiter spricht hier nicht allgemein von der Taufe, „die man als Kind

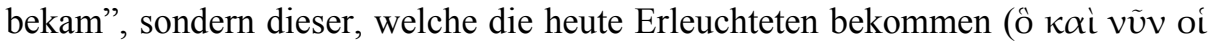

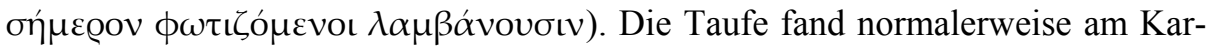
samstag statt und manchmal an anderen großen Festen, wie am Epiphaniefest. Diese Textänderung widerspiegelt wahrscheinlich eine Praktik, die in der Zeit der Bearbeitung üblich war, d.h. dass das Fest des heiligen Demetrios für Thessaloniki ein Tag für die Taufe der dafür vorbereiteten Menschen war. Ein ähnlicher Hinweis ist aus anderen Quellen nicht bekannt.

Die zweite Stelle betrifft die eingeschobene Rede von Joseph gegen den Kaiser und seine ikonoklastische Haltung. Kurtz versteht diese Polemik als gegen Leon III. gerichtet, ${ }^{22}$ es scheint mir aber unwahrscheinlich, dass sich Joseph so scharf und so direkt gegen eine Politik wendet, die einerseits nicht mehr aktuell war und andererseits von jemandem betrieben ist, der schon seit ungefähr 70 Jahren verstorben war. Es wäre m.E. logischer, dass Joseph gegen den Kaiser seiner Zeit spricht, d.h. Leon V., eine Annahme, die zu einer Datierung der Rede in die zweite Phase der Amtszeit von Joseph (811-814) führt. ${ }^{23}$ Dagegen datiert Kurtz das Enkomion in die Jahre 803-809, einerseits weil Joseph ,seine Absetzung und Wiedereinsetzung mit keinem Worte berührt" und andererseits wegen seiner Aussage, dass Friede dank der Frömmigkeit der Regierenden herrscht: $\varepsilon \grave{\imath} \gamma \dot{\alpha} \varrho \kappa \alpha \grave{\imath} \mu \grave{\eta}$

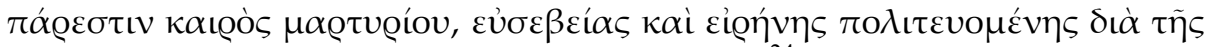

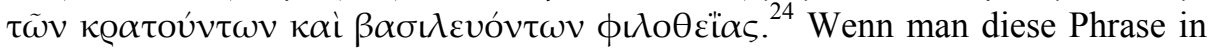
ihrem Kontext liest, kann man sie auch als eine allgemein geltende Aussage betrachten. Wenn nicht, dann darf sie als ein Hinweis für die Datierung des Enkomions vor dem Ausbruch der Krise in den Beziehungen Leons V. mit dem Patriarchen Nikephoros und den Bilderverehrern im Jahre 815 betrachtet werden. Eine solche Interpretation führt zu einer Datierung des Enkomions im Oktober 814. Die Kommission der Bischöfe, die sich im Auftrag von Leon V. mit dem Problem der Bilderverehrung beschäftigte, hatte im Dezember 814 ihre Arbeit abgeschlos-

21 Paris. 1517, f. 252v.

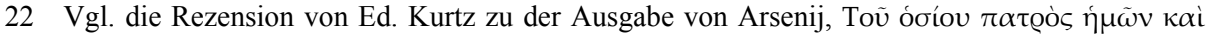

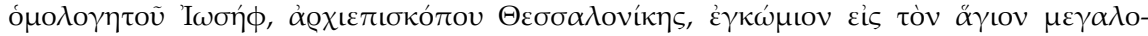

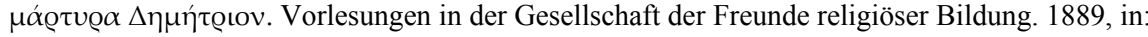
BZ 2 (1893) 631-632.

23 Die feste Anbindung der antikonoklastischen Apostrophierung im Text des Enkomions, lässt nicht die Annahme zu, dass es sich hier um eine Interpolation handelt.

24 Kurtz (s. Anm. 22), 632. 
sen. ${ }^{25}$ Obwohl nach dem Scriptor Incertus die Kommission bis dahin vertraulich gearbeitet hat, ließ die Politik von Leon V. solche Befürchtungen zu. ${ }^{26}$ Eine Datierung mit Oktober 815 ist auszuschließen, weil nach der ikonoklastischen Synode vom April 815 Joseph wahrscheinlich nicht mehr in Thessaloniki war. ${ }^{27}$

Im Mosqu. 162 (Vlad. 380) ist der Angriff wesentlich direkter als im Paris. 1517. Es ist eindeutig, dass, während der Mosqu. 162 (Vlad. 380) den ursprünglichen Text behalten hat, der Bearbeiter, dessen Text der Paris. 1517 überliefert, auf die direkte Polemik verzichtet, die seit langer Zeit ohne Gegenstand geblieben war. $^{28}$

\begin{tabular}{|c|c|}
\hline Mosqu. 162 (Vlad. 380), f. 82v-83 & Paris. 1517, f. 253 \\
\hline 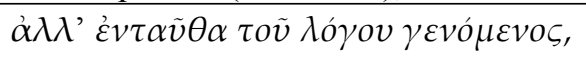 & 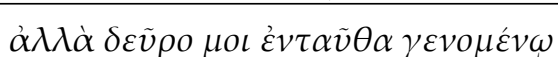 \\
\hline$\tau \dot{\eta} v \sigma \dot{\eta} v \sigma \tau \eta \lambda \iota \tau \varepsilon \dot{v} \sigma \omega \dot{\alpha} \sigma \varepsilon \beta \varepsilon\llcorner\alpha v$ & \\
\hline 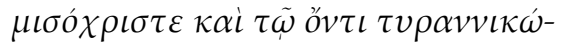 & \\
\hline$\tau \alpha \tau \varepsilon \beta \alpha \sigma \iota \lambda \varepsilon \tilde{v}, \beta \alpha \sigma \iota \lambda \varepsilon v o ́ \mu \varepsilon v \varepsilon$ vंтò $\tau o \tilde{v}$ & \\
\hline$\pi o v \eta \rho \circ \tilde{v} \kappa \alpha i \tau \tilde{i} \alpha \hat{v} \tau o \tilde{v} \pi \lambda \dot{\alpha} v \eta \kappa \alpha i$ & \\
\hline 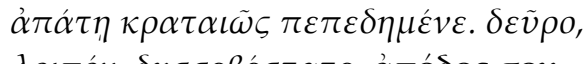 & \\
\hline 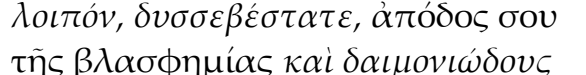 & 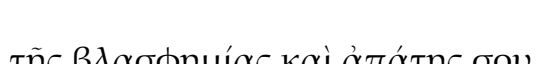 \\
\hline 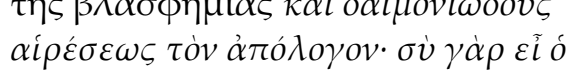 & 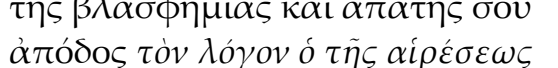 \\
\hline 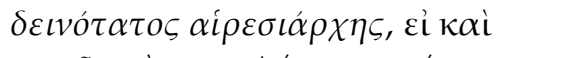 & 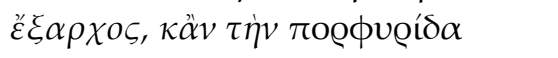 \\
\hline 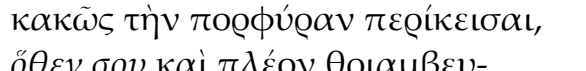 & 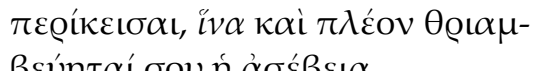 \\
\hline 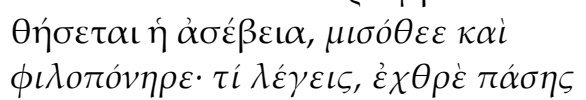 & \\
\hline 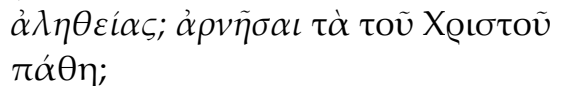 & $\dot{\alpha} \varrho v \tilde{\eta} \tau \dot{\alpha} X \rho \iota \sigma \tau o \tilde{v} \tau \alpha \dot{\alpha} \theta \eta$ \\
\hline
\end{tabular}

25 Korres (s. Anm. 7), 108.

26 Scriptor Incertus. Testo critico, traduzione e note a cura di F. Iadevaia, Messina 1987, 61.326-

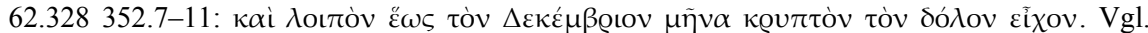
auch Korres (s. Anm. 7), 108.

27 PmbZ, Nr. 3448 (401).

28 Interessant ist in diesem Zusammenhang das Beispiel der „vita retractata“ der heiligen Theodora

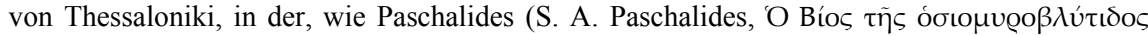

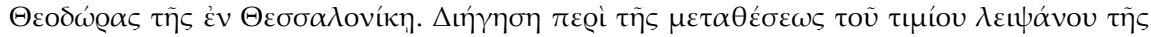

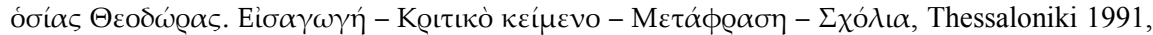
31) bemerkt hat, der am stärksten veränderte Teil der Vita die eingebaute Rede des Metropoliten von Thessaloniki Antonios gegen die Ikonoklasten ist. Es handelt sich aber hier um eine spätere Umarbeitung der aus dem 10. Jh. stammenden Vita, die möglicherweise von Nikolaos Kabasilas im 14. Jh. durchgeführt wurde; s. dazu S. Kotzabassi, Ein neues Autographon des Nikolaos Kabasilas: Der Kodex Vatic. Palat. Gr. 211, in: JÖB 53 (2003) 194. 
Das Enkomion von Joseph Studites ist sicherlich nicht das einzige Beispiel für die Umarbeitung eines hagiographischen Textes, bietet aber einen weiteren Zeugen für ein Phänomen, das auf jeden Fall weiter untersucht werden soll. 
